\title{
Invariant Lagrangians, mechanical connections and the Lagrange-Poincaré equations
}

\author{
T Mestdag ${ }^{1,2}$ and M Crampin ${ }^{2}$ \\ ${ }^{1}$ Department of Mathematics, University of Michigan, 530 Church Street, Ann \\ Arbor, MI 48109, USA \\ 2 Department of Mathematical Physics and Astronomy, Ghent University, Krijgslaan \\ 281, B-9000 Ghent, Belgium
}

\begin{abstract}
We deal with Lagrangian systems that are invariant under the action of a symmetry group. The mechanical connection is a principal connection that is associated to Lagrangians which have a kinetic energy function that is defined by a Riemannian metric. In this paper we extend this notion to arbitrary Lagrangians. We then derive the reduced Lagrange-Poincare equations in a new fashion and we show how solutions of the Euler-Lagrange equations can be reconstructed with the help of the mechanical connection. Illustrative examples confirm the theory.
\end{abstract}

AMS classification scheme numbers: 34A26, 37J15, 53C05, 70H03

Submitted to: J. Phys. A: Math. Gen. 


\section{Introduction}

The enormous importance of the concept of symmetry in a great number of applications in physics is beyond any doubt. Symmetry properties of mechanical systems in particular have been studied intensively in the last decades (see e.g. the survey in Chapter 3 of the recent monograph [8]). The bulk of the literature, however, concentrates on the Hamiltonian description of symmetric systems in which mainly the theory of Poisson manifolds plays an important role. Less well-known is the process of symmetry reduction for Lagrangian systems. When the Lagrangian is invariant under the action of a Lie group, so that we are dealing with a symmetry group of the Euler-Lagrange equations, the equations of motion can be reduced to a new set of equations with fewer unknowns. In the literature, there are in fact a lot of different paths that lead to different Lagrangian reduction theories. For example, if the configuration space is either a Lie group or a semidirect product, the distinct reduction method is called either Euler-Poincaré reduction or semi-direct product reduction. Another path is the following. In rough terminology, the invariance of the Lagrangian leads via the Noether theorem to a set of conserved quantities (the momenta). Whether or not one wants to take these conserved quantities into account in this reduction process leads to either the Routh or the Lagrange-Poincaré reduction method. For more details and some comments on the history of the above mentioned reduction theories, see e.g. [8] and [11].

In this paper, we concentrate solely on Lagrange-Poincaré reduction, and the associated reduced equations, the so-called Lagrange-Poincaré equations. The geometric framework for these equations that has been developed in e.g. [1, 10] relies heavily on methods coming from the calculus of variations. It is shown there that Hamilton's principle can be reduced to a principle on the reduced space, and that the equations of motion that follow from this reduced principle are exactly the Lagrange-Poincaré equations. In this paper, we wish to take an alternative view of Lagrange-Poincaré reduction. We will not take recourse to variations, but rather interpret the Euler-Lagrange equations as defining the integral curves of an associated second-order differential equation field on the velocity phase space. We will show that the Lagrange-Poincaré equations can be derived in a relatively straightforward fashion from the Euler-Lagrange equations, by choosing a suitable adapted frame, or equivalently by employing well-chosen quasivelocities.

Next to investigating aspects of reduction, we also focus on the inverse process, that of reconstruction. With the aid of a principal connection, for any invariant system one can reconstruct a solution of the original problem from a reduced solution. In the case of a so-called simple mechanical system (with a Lagrangian of the form $T-V$ where the kinetic energy $T$ is derived from a Riemannian metric), it is well-known that such a connection is naturally available, and it is therefore called 'the mechanical connection'. We will show in this paper that we can define for any arbitrary Lagrangian system (under a natural regularity assumption) a generalized mechanical connection, and that we can employ this connection in the process of reconstructing integral curves of the 
Euler-Lagrange field. The generalized mechanical connection is new. The construction to be found in Lewis's paper [9], while superficially similar is in fact rather different. We emphasize that the generalized mechanical connection is designed for use in the reconstruction process only and does not play a role in the reduction step.

The paper is organised as follows. In the next section we explain our approach to Lagrangian systems using adapted frames and quasi-velocities. We extend the notion of a mechanical connection to arbitrary Lagrangian systems in Section 3. In Section 4 we derive the Lagrange-Poincaré equations within our framework and in Section 5 we discuss the matter of reconstruction. We end the paper with some illustrative examples.

\section{Preliminaries}

Consider a Lagrangian function $L$, defined on the tangent bundle $\tau: T M \rightarrow M$ of some manifold $M$ (the configuration space). In terms of local coordinates $\left(x^{\alpha}, u^{\alpha}\right)$ (where the $x^{\alpha}$ are coordinates on $M$ and the $u^{\alpha}$ the corresponding fibre coordinates on $T M$ ) the Euler-Lagrange equations of $L$ are

$$
\frac{d}{d t}\left(\frac{\partial L}{\partial u^{\alpha}}\right)-\frac{\partial L}{\partial x^{\alpha}}=0
$$

We will assume that the Lagrangian is regular, which is to say that its Hessian with respect to the fibre coordinates,

$$
\frac{\partial^{2} L}{\partial u^{\alpha} \partial u^{\beta}}
$$

considered as a symmetric matrix, is everywhere non-singular. In case the Lagrangian is regular, the Euler-Lagrange equations can be written explicitly in the form of a system of differential equations $\ddot{x}^{\alpha}=\Gamma^{\alpha}(x, \dot{x})$. These equations may be thought of as defining a vector field $\Gamma$ on $T M$, a second-order differential equation field, namely

$$
\Gamma=u^{\alpha} \frac{\partial}{\partial x^{\alpha}}+\Gamma^{\alpha} \frac{\partial}{\partial u^{\alpha}}
$$

we call this the Euler-Lagrange field of $L$. In fact when $L$ is regular the Euler-Lagrange field is uniquely determined by the fact that it is a second-order differential equation field and satisfies

$$
\Gamma\left(\frac{\partial L}{\partial u^{\alpha}}\right)-\frac{\partial L}{\partial x^{\alpha}}=0
$$

In this paper we will work with regular Lagrangians and their Euler-Lagrange fields, determined by the version of the Euler-Lagrange equations given immediately above. However, we will often not use coordinates; instead, we will express everything in terms of certain convenient local bases of vector fields. We must therefore explain how to modify the formulation above to take account of this difference.

There are two canonical ways of lifting a vector field $Z$ on $M$ to one on $T M$. Firstly, the vector field on $T M$ with the property that its flow is tangent to the flow of $Z$ on $M$ is called the complete lift of $Z$, and is denoted by $Z^{\mathrm{C}}$. In terms of coordinates

$$
Z^{\mathrm{C}}=Z^{\alpha} \frac{\partial}{\partial x^{\alpha}}+\frac{\partial Z^{\beta}}{\partial x^{\alpha}} u^{\alpha} \frac{\partial}{\partial u^{\beta}}, \quad \text { where } Z=Z^{\alpha}(x) \frac{\partial}{\partial x^{\alpha}} .
$$


The second canonical way of lifting the vector field $Z$ from $M$ to $T M$ is the vertical lift:

$$
Z^{\mathrm{v}}=Z^{\alpha} \frac{\partial}{\partial u^{\alpha}}
$$

$Z^{\mathrm{v}}$ is tangent to the fibres of the projection $\tau: T M \rightarrow M$, and on the fibre over $m \in M$ it coincides with the constant vector field $Z_{m}$. In general, we have $T \tau \circ Z^{\mathrm{C}}=Z \circ \tau$ while $T \tau \circ Z^{\mathrm{v}}=0$ (regarding the vector fields as sections of the appropriate bundles). The Lie brackets of complete and vertical lifts of vector fields $Y$ and $Z$ on $M$ are given by $\left[Y^{\mathrm{C}}, Z^{\mathrm{C}}\right]=[Y, Z]^{\mathrm{C}},\left[Y^{\mathrm{C}}, Z^{\mathrm{v}}\right]=[Y, Z]^{\mathrm{V}}$ and $\left[Y^{\mathrm{v}}, Z^{\mathrm{v}}\right]=0$. It is worth emphasising that although the map $Z \mapsto Z^{\mathrm{V}}$ is $C^{\infty}(M)$-linear, the map $Z \mapsto Z^{\mathrm{C}}$ is only $\mathcal{R}$-linear: in fact for $f \in C^{\infty}(M)$

$$
(f Z)^{\mathrm{C}}=f Z^{\mathrm{C}}+\dot{f} Z^{\mathrm{v}}
$$

where $\dot{f}$ is the so-called total derivative of $f$, given in coordinates by

$$
\dot{f}=u^{\alpha} \frac{\partial f}{\partial x^{\alpha}}
$$

(a function on $T M$ ). More details on all of this can be found in e.g. [5].

Now take a local basis of vector fields on $M$, say $\left\{Z_{\alpha}\right\}$. Then $\left\{Z_{\alpha}^{\mathrm{C}}, Z_{\alpha}^{\mathrm{v}}\right\}$ is a local basis of vector fields on $T M$. Moreover, we can introduce new fibre coordinates $v^{\alpha}$ on $T M$, adapted to the local basis $\left\{Z_{\alpha}\right\}$, as follows: for any tangent vector $v_{m} \in T_{m} M$, the $v^{\alpha}$ are the components of $v$ with respect to the basis $Z_{\alpha}(m)$, that is, $v_{m}=v^{\alpha} Z_{\alpha}(m)$. Such fibre coordinates are called quasi-velocities.

From the coordinate expressions of the vertical and complete lifts above it is easy to see that if $\left\{Z_{\alpha}\right\}$ is such a basis then the equations

$$
\Gamma\left(Z_{\alpha}^{\mathrm{V}}(L)\right)-Z_{\alpha}^{\mathrm{C}}(L)=0
$$

are equivalent to the Euler-Lagrange equations. Moreover, the fact that $\Gamma$ is a secondorder differential equation field means that it takes the form

$$
\Gamma=v^{\alpha} Z_{\alpha}^{\mathrm{C}}+\hat{\Gamma}^{\alpha} Z_{\alpha}^{\mathrm{v}}
$$

where the $v^{\alpha}$ are the quasi-velocities corresponding to the basis $\left\{Z_{\alpha}\right\}$.

We will also need a coordinate-independent version of the Hessian. The Hessian of $L$ at $u \in T M$ is the symmetric bilinear form $g$ on $T_{m} M, m=\tau(u)$, given by $g(v, w)=v^{\mathrm{v}}\left(w^{\mathrm{v}}(L)\right)$, where the vertical lifts are to $u$. Alternatively we can regard $g$ as operating on pairs of vector fields $Y, Z$ on $M$ : then $g(Y, Z)$ is a function on $T M$, depending bilinearly on its arguments, such that $g(Y, Z)=Y^{\mathrm{v}}\left(Z^{\mathrm{v}}(L)\right)$; the fact that it is symmetric in $Y$ and $Z$ is a consequence of the bracket relation $\left[Y^{\mathrm{V}}, Z^{\mathrm{v}}\right]=0$ stated earlier.

We turn now to the question of symmetries and invariance.

Throughout the paper we will assume that the configuration space $M$ of the Lagrangian system comes equipped with a free and proper left action $\psi^{M}: G \times M \rightarrow M$ of a group $G$, which eventually will be the symmetry group of the Lagrangian system under consideration. In taking the group to act to the left we follow the convention of [10]; changing to a right action (as is the standard in e.g. [7]) has the effect only of a sign 
change in some of our results and formulae. The projection $\pi^{M}: M \rightarrow M / G$ on the set of equivalence classes gives $M$ the structure of a principal fibre bundle. The action on $M$ induces an action $\psi_{g}^{T M}=T \psi_{g}^{M}$ on the tangent manifold $T M$, so $\pi^{T M}: T M \rightarrow T M / G$ is also a principal fibre bundle.

A tensor field $t$ on $M$ is invariant under the action of $G$ if for all $g \in G$,

$$
t_{\psi_{g}^{M} m}\left(\psi_{g}^{T^{*} M} \alpha_{m}, \ldots, \psi_{g}^{T M} v_{m}, \ldots\right)=t_{m}\left(\alpha_{m}, \ldots, v_{m}, \ldots\right), \quad \alpha_{m} \in T_{m}^{*} M, v_{m} \in T_{m} M
$$

where $\left\langle\psi_{g}^{T^{*} M} \alpha_{m}, \psi_{g}^{T M} v_{m}\right\rangle=\left\langle\alpha_{m}, v_{m}\right\rangle$. For convenience we will assume that the group $G$ is connected. Then the invariance property is equivalent to the infinitesimal condition $\mathcal{L}_{\tilde{\xi}} t=0$ for all $\xi \in \mathfrak{g}$ (the Lie algebra of $G$ ), where $\tilde{\xi}$ on $M$ is the fundamental vector field associated with $\xi$. The proof of the equivalence relies on the fact that the flow of $\tilde{\xi}$ on $M$ is $\psi_{t}: m \mapsto \psi_{\exp (t \xi)}^{M} m$. In particular, a function $f$ on $M$ is invariant if and only if it satisfies $\tilde{\xi}(f)=0$, and a vector field $X$ on $M$ is invariant if and only if $[\tilde{\xi}, X]=0$, for all $\xi \in \mathfrak{g}$.

A tensor field on $T M$ is invariant if and only if all of its Lie derivatives by fundamental vector fields of the induced action on $T M$ vanish. The flow of such a vector field is given by $v_{m} \mapsto T \psi_{\exp (t \xi)}^{M} v_{m}$ for some $\xi \in \mathfrak{g}$, and is therefore tangent to $m \rightarrow \psi_{\exp (t \xi)}^{M} m$, the flow of the vector field $\tilde{\xi}$ on $M$. That is to say, the fundamental vector field corresponding to $\xi \in \mathfrak{g}$ of the action of $G$ on $T M$ is just $\tilde{\xi}^{\mathrm{C}}$.

For the remainder of the paper we will suppose that $L$ is a Lagrangian function on $T M$ invariant under the induced action of a connected group $G$ on $T M$, so that $L\left(\psi_{g}^{T M} v\right)=L(v)$, or equivalently $\tilde{\xi}^{\mathrm{C}}(L)=0$ for all $\xi \in \mathfrak{g}$.

We will work with a local basis $\left\{\tilde{E}_{a}, X_{i}\right\}$ of vector fields on $M$ which is adapted to the bundle structure $M \rightarrow M / G$ in the following way. The vector fields $\tilde{E}_{a}$ are the fundamental vector fields corresponding to a basis $\left\{E_{a}\right\}$ of $\mathfrak{g}$; the $X_{i}$ are the $G$-invariant horizontal lifts of a coordinate basis of vector fields on $M / G$, where the horizontal lift is associated with some principal connection $\omega$ on $M \rightarrow M / G$. The quasi-velocities corresponding to such a basis will be denoted by $\left(v^{a}, v^{i}\right)$, so that for every tangent vector $v_{m}, v_{m}=v^{a} \tilde{E}_{a}(m)+v^{i} X_{i}(m)$.

It will also be convenient to have a basis $\left\{\hat{E}_{a}, X_{i}\right\}$ that consists only of invariant vector fields. Let $U \subset M / G$ be an open set over which $M$ is locally trivial. The projection $\pi^{M}$ is locally given by projection onto the first factor in $U \times G \rightarrow U$, and the (left) action of $G$ by $\psi_{g}^{M}(x, h)=(x, g h)$. The vector fields on $M$ defined by

$$
\hat{E}_{a}:(x, g) \mapsto\left(\widetilde{\operatorname{ad}_{g} E_{a}}\right)(x, g)=\psi_{g}^{T M}\left(\tilde{E}_{a}(x, e)\right)
$$

are invariant. The relation between the sets $\left\{\hat{E}_{a}\right\}$ and $\left\{\tilde{E}_{a}\right\}$ can be expressed as

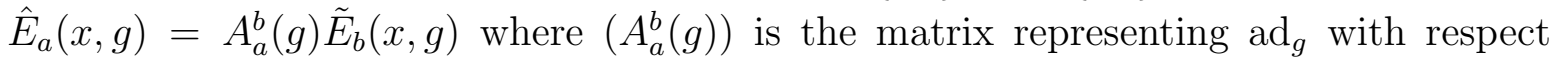
to the basis $\left\{E_{a}\right\}$ of $\mathfrak{g}$. In particular, $A_{a}^{b}(e)=\delta_{a}^{b}$. The quasi-velocities corresponding to the basis $\left\{\hat{E}, X_{i}\right\}$ will be denoted by $\left(w^{a}, v^{i}\right)$, where in fact $v^{a}=A_{b}^{a} w^{b}$.

The left-invariant vector fields $\hat{E}_{a}$ satisfy the bracket relations $\left[\hat{E}_{a}, \hat{E}_{b}\right]=C_{a b}^{c} \hat{E}_{c}$ where the $C_{a b}^{c}$ are the structure constants of the Lie algebra (so that the Lie algebra bracket satisfies $\left.\left[E_{a}, E_{b}\right]=C_{a b}^{c} E_{c}\right)$. For the fundamental vector fields, on the other hand, we 
have $\left[\tilde{E}_{a}, \tilde{E}_{b}\right]=-C_{a b}^{c} \tilde{E}_{c}$. Other Lie brackets of basis vector fields are: $\left[X_{i}, \tilde{E}_{a}\right]=0$ by invariance, whence $\left[X_{i}, \hat{E}_{a}\right]=X_{i}\left(A_{a}^{c}\right) \bar{A}_{c}^{b} \hat{E}_{b}=\Upsilon_{i a}^{b} \hat{E}_{b}$ say, where $\left(\bar{A}_{a}^{b}\right)$ is the matrix inverse to $A_{a}^{b}$; and $\left[X_{i}, X_{j}\right]=K_{i j}^{a} \hat{E}_{a}$, where the $K_{i j}^{a}$ are the components of the curvature of $\omega$, regarded as a $\mathfrak{g}$-valued tensor field.

By expressing the fact that the vector fields $\hat{E}_{a}$ are invariant in the form $\left[\tilde{E}_{b}, \hat{E}_{a}\right]=0$ we find that

$$
\tilde{E}_{b}\left(A_{a}^{c}\right)-A_{a}^{d} C_{b d}^{c}=0 .
$$

With respect to the basis $\left\{\hat{E}_{a}, X_{i}\right\}$ the Euler-Lagrange field is of the form

$$
\Gamma=w^{a} \hat{E}_{a}^{\mathrm{C}}+v^{i} X_{i}^{\mathrm{C}}+\Gamma^{a} \hat{E}_{a}^{\mathrm{V}}+\Gamma^{i} X_{i}^{\mathrm{V}} .
$$

The functions $\Gamma^{i}$ and $\Gamma^{a}$ can be determined from the Euler-Lagrange equations

$$
\begin{aligned}
& \Gamma\left(X_{i}^{\mathrm{V}}(L)\right)-X_{i}^{\mathrm{C}}(L)=0 \\
& \Gamma\left(\hat{E}_{b}^{\mathrm{V}}(L)\right)-\hat{E}_{b}^{\mathrm{C}}(L)=0 .
\end{aligned}
$$

We will now deduce from the assumed invariance of $L$ that the Euler-Lagrange field $\Gamma$ is invariant, using this machinery. That is to say, we will show that if $\tilde{E}_{a}^{\mathrm{C}}(L)=0$ for all $a$, then $\left[\tilde{E}_{a}^{\mathrm{C}}, \Gamma\right]=0$ also. One easily verifies that the quasi-velocities corresponding to an invariant basis are invariant (and in any case the derivation is given in full later); it follows that

$$
\left[\tilde{E}_{b}^{\mathrm{C}}, \Gamma\right]=\tilde{E}_{b}^{\mathrm{C}}\left(\Gamma^{i}\right) X_{i}^{\mathrm{V}}+\tilde{E}_{b}^{\mathrm{C}}\left(\Gamma^{a}\right) \hat{E}_{a}^{\mathrm{V}} .
$$

From the above Euler-Lagrange equations it easily follows that

$$
\begin{aligned}
0 & =\tilde{E}_{b}^{\mathrm{C}}\left(\Gamma\left(X_{i}^{\mathrm{V}}(L)\right)\right)-\tilde{E}_{b}^{\mathrm{C}}\left(X_{i}^{\mathrm{C}}(L)\right) \\
& =\left[\tilde{E}_{b}^{\mathrm{C}}, \Gamma\right]\left(X_{i}^{\mathrm{V}}(L)\right)+\Gamma\left(\tilde{E}_{b}^{\mathrm{C}}\left(X_{i}^{\mathrm{V}}(L)\right)\right)-\left[\tilde{E}_{b}^{\mathrm{C}}, X_{i}^{\mathrm{C}}\right](L)-X_{i}^{\mathrm{C}}\left(\tilde{E}_{b}^{\mathrm{C}}(L)\right) \\
& =\left[\tilde{E}_{b}^{\mathrm{C}}, \Gamma\right]\left(X_{i}^{\mathrm{V}}(L)\right)+\Gamma\left(\left[\tilde{E}_{b}^{\mathrm{C}}, X_{i}^{\mathrm{V}}\right](L)\right)+\Gamma\left(X_{i}^{\mathrm{V}}\left(\tilde{E}_{b}^{\mathrm{C}}(L)\right)\right) \\
& =\left[\tilde{E}_{b}^{\mathrm{C}}, \Gamma\right]\left(X_{i}^{\mathrm{V}}(L)\right) .
\end{aligned}
$$

Likewise, $\left[\tilde{E}_{b}^{\mathrm{C}}, \Gamma\right]\left(\hat{E}_{c}^{\mathrm{V}}(L)\right)=0$. From the expression for $\left[\tilde{E}_{b}^{\mathrm{C}}, \Gamma\right]$ we obtain

$$
\tilde{E}_{b}^{\mathrm{C}}\left(\Gamma^{i}\right) g\left(X_{i}, X_{j}\right)+\tilde{E}_{b}^{\mathrm{C}}\left(\Gamma^{a}\right) g\left(\hat{E}_{a}, X_{j}\right)=0=\tilde{E}_{b}^{\mathrm{C}}\left(\Gamma^{i}\right) g\left(X_{i}, \hat{E}_{c}\right)+\tilde{E}_{b}^{\mathrm{C}}\left(\Gamma^{a}\right) g\left(\hat{E}_{a}, \hat{E}_{c}\right) .
$$

Now at any point $u$ of $T M$ we may regard $\left[\tilde{E}_{b}^{\mathrm{C}}, \Gamma\right]$ as the vertical lift of some vector $w \in T_{m} M, m=\tau(u)$. From the last displayed equations we conclude that $g\left(w, X_{j}(m)\right)=0=g\left(w, \hat{E}_{c}(m)\right)$. The required conclusion that $w=0$ follows from the assumed non-singularity of $g$. Notice that it follows that $\tilde{E}_{b}^{\mathrm{C}}\left(\Gamma^{i}\right)=0$ and $\tilde{E}_{b}^{\mathrm{C}}\left(\Gamma^{a}\right)=0$.

\section{The mechanical connection}

A connection on $\pi^{M}: M \rightarrow M / G$ is a left splitting $\omega$ of the short exact sequence

$$
0 \rightarrow M \times \mathfrak{g} \rightarrow T M \stackrel{T \pi^{M}}{\rightarrow}\left(\pi^{M}\right)^{*} T(M / G) \rightarrow 0 .
$$

We identify $M \times \mathfrak{g}$ as a subbundle of $T M$ by means of $(m, \xi) \mapsto \tilde{\xi}(m)$. We will use the symbol $\omega$ for the following two related objects: $\omega$ may be thought of as a type 
$(1,1)$ tensor field on $M$, for which, in particular, $\omega(\tilde{\xi})=\tilde{\xi}$. On the other hand, we will also use $\omega$ for its projection onto $\mathfrak{g}$; we then have $\omega(\tilde{\xi}(m))=\xi$. With the second interpretation, if $\omega$ satisfies $\omega\left(\psi_{g}^{T M} v\right)=\operatorname{ad}_{g} \omega(v)$ the connection is said to be principal. The infinitesimal version of this invariance property is that $\mathcal{L}_{\tilde{\xi}} \omega=0$, for all $\xi$, where $\omega$ is now interpreted as a $(1,1)$-tensor on $M$. The kernel of $\omega$ (in either interpretation) defines a distribution on $T M$, invariant when the connection is principal, which is called the horizontal distribution. We can also define the connection by specifying its horizontal distribution.

In the case of a so-called simple mechanical system, one can associate a principal connection with the system in a natural way. In this case the Lagrangian takes the form $L=T-V$ where $T$ is a kinetic energy function, defined by a Riemannian metric $g$ on $M$, and $V$ is a function on $M$, the potential energy. The symmetry group of the Lagrangian consists of those isometries of $g$ which leave $V$ invariant. The mechanical connection $\omega$ can be defined by taking for its horizontal subspaces the orthogonal complements of the tangent spaces to the fibres of $\pi^{M}$.

The construction of the mechanical connection relies heavily on the availability of a Riemannian metric. Now the components of the metric with respect to a coordinate basis coincide with those of the Hessian of the simple Lagrangian with respect to the fibre coordinates. However, the Hessian of an arbitrary Lagrangian does not provide a Riemannian metric on configuration space. Nevertheless, the notion of a mechanical connection may be generalized in such a way that the Hessian plays the same role in relation to the generalized mechanical connection as the metric does to the mechanical connection for a simple system. This we now show.

Recall that the Hessian of $L$ at $u \in T M$ is the symmetric bilinear form $g$ on $T_{m} M$, $m=\tau(u)$, given by $g(v, w)=v^{\mathrm{v}} w^{\mathrm{v}}(L)$. As such, it can be interpreted as a tensor field along the tangent bundle projection $\tau: T M \rightarrow M$, as we show in the following paragraphs.

A vector field along $\tau$ is a section of the pullback bundle $\tau^{*} T M \rightarrow T M$. Such a section can in an equivalent way be interpreted as a map $X: T M \rightarrow T M$ with the property that $\tau \circ X=\tau$. A vector field along $\tau$ takes the local form

$$
X=X^{\alpha}(x, u) \frac{\partial}{\partial x^{\alpha}} .
$$

A vector field $Z$ on $M$ can be interpreted as the vector field $Z \circ \tau$ along $\tau$. We will call such vector fields along $\tau$ 'basic', and use the same symbol for the vector field on $M$ and the basic vector field along $\tau$.

By taking for 1-forms along $\tau$ the sections of $\tau^{*} T^{*} M \rightarrow T M$, we can obtain in the usual manner a $C^{\infty}(T M)$-module of tensor fields along $\tau$. (For more information see e.g. $[12,15]$.) In particular, we can interpret the Hessian of a Lagrangian as the symmetric $(0,2)$ tensor field along $\tau$ given by

$$
g=g_{\alpha \beta}(x, u) d x^{\alpha} \otimes d x^{\beta}, \quad g_{\alpha \beta}=\frac{\partial^{2} L}{\partial u^{\alpha} \partial u^{\beta}} .
$$


Then as we pointed out earlier, if $X, Y$ are vector fields on $M$ then $g(X, Y)=$ $X^{\mathrm{v}}\left(Y^{\mathrm{v}}(L)\right)$.

We show now that when $L$ is invariant, $g$ is also invariant in an appropriate sense.

The action of the symmetry group $G$ on $T M$ induces an action of $G$ on $\tau^{*} T M$ by $\left(g,\left(u_{m}, v_{m}\right)\right) \mapsto\left(\psi_{g}^{T M} u_{m}, \psi_{g}^{T M} v_{m}\right)$ for $g \in G$. Then, for example, a vector field $X$ along $\tau$ is invariant if

$$
X\left(\psi_{g}^{T M} v\right)=\psi_{g}^{T M}(X(v)), \quad \forall g \in G .
$$

The infinitesimal version of this property will follow from a more general construction in [6], which we adapt to the current context.

Let $\varphi: T M \rightarrow T M$ be fibred over $f: M \rightarrow M$. Then we can extend $\varphi$ to a map $\bar{\varphi}: \tau^{*}(T M) \rightarrow \tau^{*}(T M)$, fibred over $\varphi$, as follows: for $u_{m}, v_{m} \in T M$, set

$$
\bar{\varphi}\left(u_{m}, v_{m}\right)=\left(\varphi\left(u_{m}\right), T f\left(v_{m}\right)\right)
$$

where $T f: T M \rightarrow T M$ is the tangent map to $f$. We have $\tau \circ \varphi=\tau \circ T f=f$, so $\tilde{\varphi}$ is well defined. In coordinates $\left(x^{\alpha}, u^{\alpha}, v^{\alpha}\right)$, with $\varphi(x, u)=\left(f^{\alpha}(x), \varphi^{\alpha}(x, u)\right)$, we have

$$
\bar{\varphi}(x, u, v)=\left(f^{\alpha}(x), \varphi^{\alpha}(x, u), \frac{\partial f^{\alpha}}{\partial x^{\beta}} v^{\beta}\right) .
$$

Given any vector field $X$ along $\tau$ and any fibred diffeomorphism $\varphi: T M \rightarrow T M$ we can define a new vector field along $\tau, \varphi_{\sharp} X$, by $\varphi_{\sharp} X(v)=\bar{\varphi}\left(X\left(\varphi^{-1}(v)\right)\right)$. We can thus define a Lie derivative operator of vector fields on $M$ on the set of vector fields along $\tau$ : for any vector field $Z$ on $M$ and vector field $X$ along $\tau$ set

$$
\mathcal{L}_{Z} X=\frac{d}{d t}\left(\psi_{(-t) \sharp} X\right)_{t=0},
$$

where $\psi_{t}$ is the flow of $Z^{\mathrm{C}}$. If we think of $X$ as a derivation from functions on $M$ to functions on $T M$, we have for a function $F$ on $M$

$$
\mathcal{L}_{Z} X(F)=Z^{\mathrm{C}}(X(F))-X(Z(F)) .
$$

In coordinates

$$
\mathcal{L}_{Z}\left(X^{\alpha} \frac{\partial}{\partial x^{\alpha}}\right)=\left(Z^{\beta} \frac{\partial X^{\alpha}}{\partial x^{\beta}}+\frac{\partial Z^{\beta}}{\partial x^{\gamma}} u^{\gamma} \frac{\partial X^{\alpha}}{\partial u^{\beta}}-X^{\beta} \frac{\partial Z^{\alpha}}{\partial x^{\beta}}\right) \frac{\partial}{\partial x^{\alpha}} .
$$

If $X$ is a basic vector field along $\tau$ then $\mathcal{L}_{Z} X$ is the basic vector field $[Z, X]$ along $\tau$, where the bracket is the bracket of vector fields on $M$.

The operator $\mathcal{L}_{Z}$ has all the usual properties of the Lie derivative, except that for functions $F$ on $T M, \mathcal{L}_{Z}(F X)=Z^{\mathrm{C}}(F) X+F \mathcal{L}_{Z} X$. So if we define the action of $\mathcal{L}_{Z}$ on functions by $\mathcal{L}_{Z} F=Z^{\mathrm{C}}(F)$, we can extend the action to the tensor algebra of $\tau^{*} T M$ in the usual way. In particular, for a type $(0,2)$ tensor field $g$ and vector fields $X, Y$ along $\tau$,

$$
\left(\mathcal{L}_{Z} g\right)(X, Y)=Z^{\mathrm{C}}(g(X, Y))-g\left(\mathcal{L}_{Z} X, Y\right)-g\left(X, \mathcal{L}_{Z} Y\right) .
$$

Consider the case where $X$ is an invariant vector field along $\tau$, and where $Z$ is a fundamental vector field $\tilde{\xi}$. The flow of $\tilde{\xi}$ is exactly $f_{t}: m \mapsto \psi_{\exp (t \xi)}^{M} m$. By 
definition of a complete lift, the flow of $\tilde{\xi}^{\mathrm{C}}$ is the tangent of the flow of $\tilde{\xi}$, that is $\psi_{t}: v \mapsto T \psi_{\exp (t \xi)}^{M} v=\psi_{\exp (t \xi)}^{T M} v$. Since $\left(\psi_{-t}\right)^{-1}=\psi_{t}$, we find that

$$
\left(\left(\psi_{-t}\right)_{\sharp} X\right)(v)=\bar{\psi}_{-t}\left(X\left(\psi_{t} v\right)=\left(\psi_{-t}\left(\psi_{t} v\right), T f_{-t}\left(X\left(\psi_{t} v\right)\right)\right)=(v, X(v)),\right.
$$

where we have used the invariance property of $X$ with respect to group elements of the form $\exp (t \xi)$. So $\left(\psi_{-t}\right)_{\sharp} X=X$ and we conclude that $\mathcal{L}_{\tilde{\xi}} X=0$ for all $\xi \in \mathfrak{g}$. Since $G$ is supposed to be connected, a standard argument shows that this criterion is in fact sufficient.

The vertical lift operation described earlier can easily be extended to vector fields along $\tau$. From the coordinate expression of the Lie derivative it is easy to see that $\left(\mathcal{L}_{Z} X\right)^{\mathrm{v}}=\left[Z^{\mathrm{C}}, X^{\mathrm{v}}\right]$. It follows in particular that a vector field $X$ along $\tau$ is invariant if and only if its vertical lift is invariant as a vector field on $T M$.

With a similar argument to the one for vector fields along $\tau$, we can conclude that a tensor field $g$ along $\tau$ is invariant if and only if $\mathcal{L}_{\tilde{\xi}} g=0$ for all $\xi \in \mathfrak{g}$. In fact it is enough to show that this tensor vanishes when its arguments are basic vector fields $X, Y$ along $\tau$. Let $g$ be the Hessian of an invariant Lagrangian. Recall that for $X, Y$ basic, $g(X, Y)=X^{\mathrm{v}}\left(Y^{\mathrm{v}}(L)\right)$. We have

$$
\begin{aligned}
\left(\mathcal{L}_{\tilde{\xi}} g\right)(X, Y) & =\tilde{\xi}^{\mathrm{C}}(g(X, Y))-g\left(\mathcal{L}_{\tilde{\xi}} X, Y\right)-g\left(X, \mathcal{L}_{\tilde{\xi}} Y\right) \\
& =\tilde{\xi}^{\mathrm{C}}\left(X^{\mathrm{V}} Y^{\mathrm{V}}(L)\right)-[\tilde{\xi}, X]^{\mathrm{V}} Y^{\mathrm{V}}(L)-X^{\mathrm{V}}[\tilde{\xi}, Y]^{\mathrm{v}}(L) \\
& =\left[\tilde{\xi}^{\mathrm{C}}, X^{\mathrm{V}}\right] Y^{\mathrm{V}}(L)+X^{\mathrm{v}} \tilde{\xi}^{\mathrm{C}} Y^{\mathrm{V}}(L)-[\tilde{\xi}, X]^{\mathrm{V}} Y^{\mathrm{V}}(L)-X^{\mathrm{V}}[\tilde{\xi}, Y]^{\mathrm{V}}(L) \\
& =\left[\tilde{\xi}^{\mathrm{C}}, X^{\mathrm{V}}\right] Y^{\mathrm{V}}(L)-[\tilde{\xi}, X]^{\mathrm{V}} Y^{\mathrm{V}}(L)+X^{\mathrm{V}}\left[\tilde{\xi}^{\mathrm{C}}, Y^{\mathrm{V}}\right](L)-X^{\mathrm{v}}[\tilde{\xi}, Y]^{\mathrm{V}}(L) \\
& =0,
\end{aligned}
$$

since by assumption $\tilde{\xi}^{\mathrm{C}}(L)=0$.

We turn now to the definition of the generalized mechanical connection. We first make some remarks about connections in this general context.

The short exact sequence $0 \rightarrow M \times \mathfrak{g} \rightarrow T M \rightarrow \pi^{M^{*}} T(M / G) \rightarrow 0$ extends in a natural way to an exact sequence of vector bundles over $T M$,

$$
0 \rightarrow T M \times \mathfrak{g} \rightarrow \tau^{*} T M \rightarrow\left(\pi^{M} \circ \tau\right)^{*} T(M / G) \rightarrow 0,
$$

where the first space is spanned by basic vector fields $\tilde{\xi}$ along $\tau$. We will call an invariant left splitting of this sequence an invariant connection along $\tau$. Equivalently, an invariant connection along $\tau$ is a $(1,1)$-tensor field $\omega$ along $\tau$ with the property that $\mathcal{L}_{\tilde{\xi}} \omega=0$ for all $\xi \in \mathfrak{g}$. For a basic vector field $\tilde{\eta}$ along $\tau$ we have $\omega(\tilde{\eta})=\tilde{\eta}$. A vector field $X$ along $\tau$ which satisfies $\omega(X)=0$ is said to be horizontal.

From the fact that $\omega(\tilde{\eta})=\tilde{\eta}$ it follows automatically that

$$
\left(\mathcal{L}_{\tilde{\xi}} \omega\right)(\tilde{\eta})=\omega([\tilde{\xi}, \tilde{\eta}])-[\tilde{\xi}, \omega(\tilde{\eta})]=0 .
$$

So, for invariance, the only condition we need to check is that for all horizontal vector fields $X$ along $\tau, \mathcal{L}_{\tilde{\xi}} X$ is horizontal also. In fact

$$
\left(\mathcal{L}_{\tilde{\xi}} \omega\right)(X)=\omega\left(\mathcal{L}_{\tilde{\xi}} X\right)-\mathcal{L}_{\tilde{\xi}}(\omega(X))=\omega\left(\mathcal{L}_{\tilde{\xi}} X\right)
$$

so $\mathcal{L}_{\tilde{\xi}} \omega=0$ if and only $\mathcal{L}_{\tilde{\xi}} X$ is horizontal whenever $X$ is horizontal. 
Any principal connection on $M \rightarrow M / G$ can be extended to an invariant connection along $\tau$ in a trivial way.

In the case of an invariant Lagrangian system we can define a connection along $\tau$ by adapting the construction of the mechanical connection of a simple mechanical system; the result is the generalized mechanical connection, which we denote by $\omega^{\mathrm{m}}$. A vector field $X$ along $\tau$ is horizontal with respect to $\omega^{\mathrm{m}}$ if it is in the orthogonal complement of $T M \times \mathfrak{g}$ with respect to $g$, that is if

$$
g(\tilde{\xi}, X)=0, \quad \forall \xi \in \mathfrak{g} .
$$

Of course, this defines a splitting only if we suppose that $g$ is non-singular when restricted to the set of fundamental vector fields; this will be the case in particular if $g$ is everywhere positive-definite.

We now show that $\omega^{\mathrm{m}}$ is invariant, by showing that if $X$ is a horizontal vector field along $\tau$, so is $\mathcal{L}_{\tilde{\xi}} X$ for all $\xi \in \mathfrak{g}$. For a horizontal $X$

$$
\begin{aligned}
0 & =\left(\mathcal{L}_{\tilde{\xi}} g\right)(\tilde{\eta}, X)=\tilde{\xi}^{\mathrm{C}}(g(\tilde{\eta}, X))-g([\tilde{\xi}, \tilde{\eta}], X)-g\left(\tilde{\eta}, \mathcal{L}_{\tilde{\xi}} X\right) \\
& =g([\widetilde{\xi, \eta}], X)-g\left(\tilde{\eta}, \mathcal{L}_{\tilde{\xi}} X\right) \\
& =-g\left(\tilde{\eta}, \mathcal{L}_{\tilde{\xi}} X\right),
\end{aligned}
$$

so $\mathcal{L}_{\tilde{\xi}} X$ is indeed horizontal, as claimed.

We can lift $\omega^{\mathrm{m}}$ in a natural way to define a principal connection $\Omega^{\mathrm{m}}$ on $T M \rightarrow T M / G$. The process by which we obtain $\Omega^{\mathrm{m}}$ from $\omega^{\mathrm{m}}$ is a variant of the so-called vertical lift of a principal connection $\omega$ on $\pi^{M}: M \rightarrow M / G$ to $T M \rightarrow T M / G$ : the vertical lift of $\omega$, considered here as a $\mathfrak{g}$-valued 1 -form on $M$, is just $\tau^{*} \omega$, a $\mathfrak{g}$-valued 1 -form on $T M$. It is easy to show that $\tau^{*} \omega$ is a principal connection on $\pi^{T M}: T M \rightarrow T M / G$ : see for example [2].

The vertical lift of the generalized mechanical connection $\omega^{\mathrm{m}}$ is the $\mathfrak{g}$-valued 1 -form $\Omega^{\mathrm{m}}$ on $\pi^{T M}$ defined by

$$
\Omega^{\mathrm{m}}(W)=\omega^{\mathrm{m}}\left(\tau_{*} W\right)
$$

for any vector field $W$ on $T M$; here $\tau_{*} W$ is the projection of $W$ regarded as a vector field along $\tau$. Thus a vector field $W$ on $T M$ is horizontal if $g\left(\tau_{*} W, \tilde{\eta}\right)=0$ for all $\eta \in \mathfrak{g}$, where as usual $\tilde{\eta}$ is regarded as a basic vector field along $\tau$. It is clear that the horizontal subspace of $T_{u} T M$ is complementary to the tangent to the fibre of $\pi^{T M}$, provided that $g_{u}$ is non-singular on the tangent to the fibre of $\pi^{M}$, as before. Note in particular the paradoxical-sounding fact that every vertical vector field is horizontal: to be precise, every vector field on $T M$ which is vertical with respect to the tangent bundle projection $\tau$ is horizontal with respect to the connection $\Omega^{\mathrm{m}}$. Now $\Omega^{\mathrm{m}}$ defines a principal connection if $\left[\tilde{\xi}^{\mathrm{C}}, W\right]$ is horizontal whenever $W$ is horizontal. From the definition of the generalized Lie derivative above it is easy to see that for any vector field $W$ on $T M$ we have

$$
\mathcal{L}_{Z}\left(\tau_{*} W\right)=\tau_{*}\left[Z^{\mathrm{C}}, W\right] .
$$

Then, indeed,

$$
g\left(\tau_{*}\left[\tilde{\xi}^{\mathrm{C}}, W\right], \tilde{\eta}\right)=g\left(\mathcal{L}_{\tilde{\xi}}\left(\tau_{*} W\right), \tilde{\eta}\right)=-g\left(\tau_{*} W, \mathcal{L}_{\tilde{\xi}} \tilde{\eta}\right)=-g\left(\tau_{*} W,[\tilde{\xi}, \tilde{\eta}]\right)=0 .
$$


This confirms that $\Omega^{\mathrm{m}}$ is a principal connection on $\pi^{T M}$.

The generalized mechanical connection $\omega^{\mathrm{m}}$ and its vertical lift $\Omega^{\mathrm{m}}$ are intrinsic to the invariant Lagrangian system; that is to say, it is not necessary to invoke a principal connection on $\pi^{M}: M \rightarrow M / G$ to define them. Nevertheless it is often convenient to work with a local basis of vector fields $\left\{\tilde{E}_{a}, X_{i}\right\}$ on $M$, with $X_{i}$ invariant, as described earlier; and this implicitly involves a principal connection on $\omega$ on $\pi^{M}$. In general $\omega$ will not be directly related to $\omega^{\mathrm{m}}$; but for a simple mechanical system we can take for $\omega$ the mechanical connection, in which case $\omega^{\mathrm{m}}$ is the natural extension of $\omega$ to an invariant connection along $\tau$, and $\Omega^{\mathrm{m}}$ is the vertical lift of $\omega$.

We will end this section by expressing the generalized mechanical connection and its vertical lift in terms of the basis $\left\{\tilde{E}_{a}, X_{i}\right\}$.

Let us express the components of the Hessian $g$ in terms of the basis $\left\{\tilde{E}_{a}, X_{i}\right\}$ as follows:

$$
g\left(\tilde{E}_{a}, \tilde{E}_{b}\right)=g_{a b}, \quad g\left(X_{i}, X_{j}\right)=g_{i j}, \quad g\left(X_{i}, \tilde{E}_{a}\right)=g_{i a}=g_{a i}=g\left(\tilde{E}_{a}, X_{i}\right)
$$

(in general these will be functions on $T M$, not $M$, of course). We have $g_{a b}=\tilde{E}_{a}^{\mathrm{V}}\left(\tilde{E}_{b}^{\mathrm{V}}(L)\right)$, etc. Recall that to define the generalized mechanical connection we have assumed that $\left(g_{a b}\right)$ is non-singular. If we set

$$
\bar{X}_{i}=X_{i}-g^{a b} g_{i b} \tilde{E}_{a}=X_{i}+B_{i}^{a} \tilde{E}_{a}
$$

then the $\bar{X}_{i}$ are vector fields along $\tau$ which are horizontal with respect to the generalized mechanical connection, and so

$$
\omega^{\mathrm{m}}\left(\tilde{E}_{a}\right)=\tilde{E}_{a}, \quad \omega^{\mathrm{m}}\left(\bar{X}_{i}\right)=0 .
$$

The invariance of the Hessian $g$ amounts for its coefficients to

$$
\tilde{E}_{a}^{\mathrm{C}}\left(g_{i j}\right)=0, \quad \tilde{E}_{a}^{\mathrm{C}}\left(g_{b c}\right)=C_{a b}^{d} g_{c d}+C_{a c}^{d} g_{b d}, \quad \tilde{E}_{a}^{\mathrm{C}}\left(g_{i c}\right)=C_{a c}^{d} g_{i d} .
$$

It follows that $\tilde{E}_{a}^{\mathrm{C}}\left(B_{i}^{b}\right)=B_{i}^{c} C_{c a}^{b}$. It is now easy to see that $\bar{X}_{i}$ is an invariant vector field along $\tau$ :

$$
\mathcal{L}_{\tilde{E}_{a}} \bar{X}_{i}=\mathcal{L}_{\tilde{E}_{a}} X_{i}+\tilde{E}_{a}^{\mathrm{C}}\left(B_{i}^{b}\right) \tilde{E}_{b}+B_{i}^{b} \mathcal{L}_{\tilde{E}_{a}} \tilde{E}_{b}=\left[\tilde{E}_{a}, X_{i}\right]+C_{c a}^{b} B_{i}^{c} \tilde{E}_{b}+B_{i}^{b}\left[\tilde{E}_{a}, \tilde{E}_{b}\right]=0 .
$$

Furthermore, let us define vector fields

$$
\bar{X}_{i}^{\mathrm{C}}=X_{i}^{\mathrm{C}}+B_{i}^{a} \tilde{E}_{a}^{\mathrm{C}}
$$

on $T M$. (The notation $\bar{X}_{i}^{\mathrm{C}}$ is not intended to imply that these vector fields are complete lifts of vector fields on $M$.) One can easily verify that the vector fields $\bar{X}_{i}^{\mathrm{c}}$ are invariant: $\left[\tilde{E}_{a}^{\mathrm{C}}, \bar{X}_{i}^{\mathrm{C}}\right]=0$. For the lifted connection $\Omega^{\mathrm{m}}$ we get

$$
\Omega^{\mathrm{m}}\left(\tilde{E}_{a}^{\mathrm{C}}\right)=\tilde{E}_{a}^{\mathrm{C}}, \quad \Omega^{\mathrm{m}}\left(\tilde{E}_{a}^{\mathrm{V}}\right)=0, \quad \Omega^{\mathrm{m}}\left(\bar{X}_{i}^{\mathrm{C}}\right)=0 \quad \text { and } \quad \Omega^{\mathrm{m}}\left(X_{i}^{\mathrm{V}}\right)=0 .
$$

\section{The reduced Euler-Lagrange field}

As before, we assume that the Lagrangian $L$ is invariant under a symmetry group $G$, so that $\tilde{\xi}^{\mathrm{C}}(L)=0$. Then $L$ defines a function $l$ on $T M / G$, the reduced Lagrangian, such that $L=l \circ \pi^{T M}$, where $\pi^{T M}$ is the projection of the principal fibre bundle $T M \rightarrow T M / G$. 
We showed above that the Euler-Lagrange field $\Gamma$ is also invariant. As an invariant vector field, $\Gamma$ on $T M$ defines a $\pi^{T M}$-related reduced vector field $\check{\Gamma}$ on $T M / G$ : due to the invariance of $\Gamma$, the relation $T \pi^{T M}(\Gamma(v))=\check{\Gamma}\left(\pi^{T M}(v)\right)$ is independent of the choice of $v \in T M$ within the equivalence class of $\pi^{T M}(v) \in T M / G$.

Our aim in this section is to give an explicit expression for the reduced Euler-Lagrange field $\check{\Gamma}$ on $T M / G$ in terms of the reduced Lagrangian $l$.

From now on we will use local coordinates on $M$ defined as follows. Let $U \subset M / G$ be an open set over which $M$ is locally trivial, so that $\left(\pi^{M}\right)^{-1}(U) \simeq U \times G$, and which is a coordinate neighbourhood in $M / G$. We take coordinates $\left(x^{\alpha}\right)=\left(x^{i}, x^{a}\right)$ on a suitable open subset of $\left(\pi^{M}\right)^{-1}(U)$ (containing $\left.U \times e\right)$ such that $\left(x^{i}\right)$ are coordinates on $U,\left(x^{a}\right)$ coordinates on the fibre $G$. Then $\tilde{E}_{a}$ and $\hat{E}_{a}$ can be identified with vector fields on $G$, right- and left-invariant respectively, and so are independent of the $x^{i}$. Recall that $X_{i}$ is an invariant vector field on $M$ projecting onto $\partial / \partial x^{i}$ on $M / G$. If we set

$$
X_{i}=\frac{\partial}{\partial x^{i}}-\gamma_{i}^{b}\left(x^{i}, x^{a}\right) \hat{E}_{b}
$$

then invariance of the $X_{i}$ means that

$$
\frac{\partial \gamma_{i}^{b}}{\partial x^{a}}=0
$$

Recall that we have set $\left[X_{i}, \hat{E}_{a}\right]=\Upsilon_{i a}^{b} \hat{E}_{b}$. Then $\Upsilon_{i a}^{b}=-\gamma_{i}^{c} C_{c a}^{b}$.

An invariant vector field $W$ on $T M$ projects onto a vector field $\check{W}$ on $T M / G$, so that (as sections)

$$
T \pi^{T M} \circ W=\check{W} \circ \pi^{T M} .
$$

Furthermore, if $F$ is an invariant function on $T M$ and if $f$ is its reduction to a function on $T M / G$ then

$$
W(F)=W\left(f \circ \pi^{T M}\right)=\check{W}(f) \circ \pi^{T M} .
$$

We will now show in detail that the coordinate functions $x^{i}, v^{i}$ and $w^{a}$ (where $w^{a}$ and $v^{i}$ are the quasi-velocities corresponding to our chosen invariant basis $\left.\left\{\hat{E}_{a}, X_{i}\right\}\right)$ are invariant functions on $T M$. As a consequence, they can be used as coordinates on $T M / G$. Then the action of $W$ on these functions completely determines $\breve{W}$. We are in particular interested in the reduced fields of the invariant vector fields $X_{i}^{\mathrm{C}}, \bar{X}_{i}^{\mathrm{C}}, X_{i}^{\mathrm{v}}$ and $\hat{E}_{a}^{\mathrm{V}}$ on $T M$.

The following observation can easily be verified. For any vector field $Z$, function $f$ and 1-form $\theta$ on $M$,

$$
Z^{\mathrm{C}}(f)=Z(f), \quad Z^{\mathrm{V}}(f)=0, \quad Z^{\mathrm{C}}(\vec{\theta})=\overrightarrow{\mathcal{L}_{Z} \theta}, \quad Z^{\mathrm{V}}(\vec{\theta})=\tau^{*} \theta(Z),
$$

where $\vec{\theta}$ stands for the fibre-linear function on $T M$ defined by the 1 -form $\theta$. Note that if $\left\{Z_{\alpha}\right\}$ is a local basis of vector fields on $M$ and $\theta^{\alpha}$ the dual local basis of 1 -forms, then the quasi-velocities $v^{\alpha}$ corresponding to the given vector-field basis, regarded as functions on $T M$, are given by $v^{\alpha}=\vec{\theta}^{\alpha}$. 
Let $\left\{\varpi^{a}, \vartheta^{i}\right\}$ be the dual 1-form basis of the basis $\left\{\hat{E}_{a}, X_{i}\right\}$. Then $\vec{\varpi}^{a}=w^{a}$ and $\vec{\vartheta}^{i}=v^{i}$. We have, for example,

$$
\begin{aligned}
& \left(\mathcal{L}_{X_{i}} \varpi^{a}\right)\left(\hat{E}_{b}\right)=X_{i}\left(\delta_{b}^{a}\right)-\varpi^{a}\left(\left[X_{i}, \hat{E}_{b}\right]\right)=-\Upsilon_{i b}^{a} \\
& \left(\mathcal{L}_{X_{i}} \varpi^{a}\right)\left(X_{j}\right)=-\varpi^{a}\left(\left[X_{i}, X_{j}\right]\right)=-K_{i j}^{a},
\end{aligned}
$$

so $X_{i}^{\mathrm{C}}\left(w^{a}\right)=-K_{i j}^{a} v^{j}-\Upsilon_{i b}^{a} w^{b}$. The relevant derivatives, obtained in the case of the quasi-velocities by similar calculations, are

$$
\begin{aligned}
& X_{i}^{\mathrm{C}}\left(x^{j}\right)=\delta_{i}^{j}, \quad X_{i}^{\mathrm{C}}\left(v^{j}\right)=0, \quad X_{i}^{\mathrm{C}}\left(w^{a}\right)=-K_{i j}^{a} v^{j}-\Upsilon_{i b}^{a} w^{b}, \\
& X_{i}^{\mathrm{V}}\left(x^{j}\right)=0, \quad X_{i}^{\mathrm{V}}\left(v^{j}\right)=\delta_{i}^{j}, \quad X_{i}^{\mathrm{V}}\left(w^{a}\right)=0, \\
& \hat{E}_{a}^{\mathrm{C}}\left(x^{j}\right)=0, \quad \hat{E}_{a}^{\mathrm{C}}\left(v^{i}\right)=0, \quad \hat{E}_{a}^{\mathrm{C}}\left(w^{b}\right)=\Upsilon_{i a}^{b} v^{i}+C_{a c}^{b} w^{c}, \\
& \hat{E}_{a}^{\mathrm{V}}\left(x^{j}\right)=0, \quad \hat{E}_{a}^{\mathrm{V}}\left(v^{i}\right)=0, \quad \hat{E}_{a}^{\mathrm{V}}\left(w^{b}\right)=\delta_{a}^{b}, \\
& \tilde{E}_{a}^{\mathrm{C}}\left(x^{j}\right)=0, \quad \tilde{E}_{a}^{\mathrm{C}}\left(v^{i}\right)=0, \quad \tilde{E}_{a}^{\mathrm{C}}\left(w^{b}\right)=0, \\
& \tilde{E}_{a}^{\mathrm{V}}\left(x^{j}\right)=0, \quad \tilde{E}_{a}^{\mathrm{V}}\left(v^{i}\right)=0, \quad \tilde{E}_{a}^{\mathrm{V}}\left(w^{b}\right)=\bar{A}_{a}^{b} .
\end{aligned}
$$

From the penultimate row we conclude that $x^{i}, v^{i}$ and $w^{a}$ are invariant. Therefore

$$
\begin{aligned}
& T \pi^{T M} \circ X_{i}^{\mathrm{C}}=\left(\frac{\partial}{\partial x^{i}}+\left(-K_{i j}^{a} v^{j}-\Upsilon_{i b}^{a} w^{b}\right) \frac{\partial}{\partial w^{a}}\right) \circ \pi^{T M}=T \pi^{T M} \circ \bar{X}_{i}^{\mathrm{C}}, \\
& T \pi^{T M} \circ X_{i}^{\mathrm{V}}=\frac{\partial}{\partial v^{j}} \circ \pi^{T M}, \\
& T \pi^{T M} \circ \hat{E}_{a}^{\mathrm{C}}=\left(\Upsilon_{i a}^{b} v^{i}+C_{a c}^{b} w^{c}\right) \frac{\partial}{\partial w^{b}} \circ \pi^{T M}, \quad T \pi^{T M} \circ \hat{E}_{a}^{\mathrm{V}}=\frac{\partial}{\partial w^{a}} \circ \pi^{T M} .
\end{aligned}
$$

As we noted earlier the Euler-Lagrange field is invariant. The Euler-Lagrange equations, in terms of the invariant basis $\left\{\hat{E}_{a}, X_{i}\right\}$, are

$$
\begin{aligned}
& \Gamma\left(X_{i}^{\mathrm{V}}(L)\right)-X_{i}^{\mathrm{C}}(L)=0 \\
& \Gamma\left(\hat{E}_{b}^{\mathrm{V}}(L)\right)-\hat{E}_{b}^{\mathrm{C}}(L)=0 .
\end{aligned}
$$

Using invariance we see that

$$
\Gamma\left(X_{i}^{\mathrm{V}}(L)\right)=\Gamma\left(\check{X}_{i}^{\mathrm{V}}(l) \circ \pi^{T M}\right)=\check{\Gamma}\left(\check{X}_{i}^{\mathrm{V}}(l)\right) \circ \pi^{T M},
$$

and similarly for the other terms in the Euler-Lagrange equations. These may therefore be written, entirely in terms of vector fields and functions on $T M / G$, as

$$
\begin{aligned}
& \check{\Gamma}\left(\check{X}_{i}^{\mathrm{V}}(l)\right)-\check{X}_{i}^{\mathrm{C}}(l)=0 \\
& \check{\Gamma}\left(\check{E}_{b}^{\mathrm{V}}(l)\right)-\check{E}_{b}^{\mathrm{C}}(l)=0,
\end{aligned}
$$

where $\check{E}_{b}^{\mathrm{v}}$ and $\check{E}_{b}^{\mathrm{C}}$ are the projections onto $T M / G$ of the invariant vector fields $\hat{E}_{b}^{\mathrm{v}}$ and $\hat{E}_{b}^{\mathrm{C}}$. We can now employ the coordinate expressions for the reduced vector fields $\check{X}_{i}^{\mathrm{V}}$ etc. obtained earlier to express these equations as

$$
\begin{aligned}
& \check{\Gamma}\left(\frac{\partial l}{\partial v^{i}}\right)-\frac{\partial l}{\partial x^{i}}=\left(K_{i k}^{a} v^{k}+\Upsilon_{i b}^{a} w^{b}\right) \frac{\partial l}{\partial w^{a}} \\
& \check{\Gamma}\left(\frac{\partial l}{\partial w^{a}}\right)=\left(\Upsilon_{i a}^{b} v^{i}+C_{a c}^{b} w^{c}\right) \frac{\partial l}{\partial w^{b}} .
\end{aligned}
$$

Recall that, with respect to the basis $\left\{X_{i}, \hat{E}_{a}\right\}$, the Euler-Lagrange field is of the form $\Gamma=w^{a} \hat{E}_{a}^{\mathrm{C}}+v^{i} X_{i}^{\mathrm{C}}+\Gamma^{a} \hat{E}_{a}^{\mathrm{V}}+\Gamma^{i} X_{i}^{\mathrm{V}}$. Given that $C_{a b}^{c} w^{a} w^{b}=0$ and $K_{i j}^{a} v^{i} v^{j}=0$, the 
coordinate expression of the reduced field is simply $\check{\Gamma}=v^{i} \partial / \partial x^{i}+\Gamma^{i} \partial / \partial v^{i}+\Gamma^{a} \partial / \partial w^{a}$; as we observed earlier, $\Gamma^{i}$ and $\Gamma^{a}$ are invariant, so may be considered as functions on $T M / G$. The above equations, usually written in the form

$$
\begin{aligned}
& \frac{d}{d t}\left(\frac{\partial l}{\partial v^{i}}\right)-\frac{\partial l}{\partial x^{i}}=\left(K_{i k}^{a} v^{k}+\Upsilon_{i b}^{a} w^{b}\right) \frac{\partial l}{\partial w^{a}} \\
& \frac{d}{d t}\left(\frac{\partial l}{\partial w^{a}}\right)=\left(\Upsilon_{i a}^{b} v^{i}+C_{a c}^{b} w^{c}\right) \frac{\partial l}{\partial w^{b}},
\end{aligned}
$$

are the so-called Lagrange-Poincaré equations (see e.g. [1]).

The specific structure of the manifold and the symmetry group at hand can lead to some interesting subcases. For example, if the symmetry group $G$ happens to be Abelian, all terms containing the structure coefficients of the Lie algebra vanish. From the last equation we then obtain that the 'momentum' $\partial l / \partial w^{a}$ is constant, let's say $\mu_{a}$. If moreover the matrix $\left(\partial^{2} l / \partial w^{a} \partial w^{b}\right)$ is non-singular, the relation $\partial l / \partial w^{a}=\mu_{a}$ can be rewritten in the form $w^{a}=\rho^{a}(x, v)$. With that, the first equation becomes a second order differential equation in the variables $x^{i}$. By introducing Routh's (reduced) function

$$
\mathcal{R}^{\mu}(x, v)=l(x, v, \rho(x, v))-\mu_{a} \rho^{a}(x, v)
$$

the first equation can equivalently be rewritten as

$$
\frac{d}{d t}\left(\frac{\partial \mathcal{R}^{\mu}}{\partial v^{i}}\right)-\frac{\partial \mathcal{R}^{\mu}}{\partial x^{i}}=K_{i k}^{a} v^{k} \mu_{a}
$$

This equation is known as Routh's (reduced) equation for an Abelian symmetry group. Routh's reduction process can be extended to non-Abelian symmetry groups. However, the extension requires that the two steps in the process above (first reduction and then restriction to a level set of momentum) be alternated. For more details, see [3] and [10]. There is no obvious way to relate the Lagrange-Poincaré equations to the reduced Routh equations in the case of a non-Abelian symmetry group.

Another interesting case occurs when the manifold $M$ is a product $N \times G$. Then, we can choose the connection to be trivial and all terms involving connection coefficients will vanish. Finally, if the manifold is in fact the Lie group $G$, the equations

$$
\frac{d}{d t}\left(\frac{\partial l}{\partial w^{a}}\right)=C_{a c}^{b} w^{c} \frac{\partial l}{\partial w^{b}}
$$

are known as the Euler-Poincaré equation, see e.g. [10].

\section{Reconstruction}

Now that we have derived the reduced form of the Euler-Lagrange equations it remains to consider the problem of reconstruction: suppose we can find a solution of these equations, that is, an integral curve of $\check{\Gamma}$, how do we reconstruct from it a solution of the original equations, that is, an integral curve of $\Gamma$ ?

There is in fact a standard method for reconstructing integral curves of an invariant vector field from reduced data, which makes use of connection theory. Let $\pi: M \rightarrow B$ 
be a principal fibre bundle with group $G$. An invariant vector field $X$ on $M$ defines a $\pi$-related reduced vector field $\check{X}$ on $B$. Let us suppose that $M$ is equipped with a principal connection $\omega$. Let $\check{v}(t)$ be an integral curve of $\check{X}$ (in $B$ ). Let $m$ be a point of $M$ in the fibre over $\check{v}(0)$ : we aim to find the integral curve of $X$ though $m$. We first lift $\check{v}(t)$ to $M$ by using the connection to form its horizontal lift through $m, \check{v}^{\mathrm{H}}(t)$ : this is the unique curve in $M$ projecting onto $\check{v}$ such that $\omega\left(\check{v}^{\mathrm{H}}\right)=0$ and $\check{v}^{\mathrm{H}}(0)=m$. Now let $v(t)$ be the integral curve of $X$ through $m$. Since $v(t)$ also projects onto $\check{v}(t)$ there is a curve $t \mapsto g(t) \in G$, with $g(0)=e$, such that $v(t)=\psi_{g(t)}^{M} \check{v}^{\mathrm{H}}(t)$. On differentiating this equation we obtain

$$
\dot{v}=\psi_{g}^{T M}\left(\widetilde{\vartheta(\dot{g})} \circ \check{v}^{\mathrm{H}}+\check{\check{v}}^{\mathrm{H}}\right)
$$

where $\vartheta$ is the Maurer-Cartan form of $G$ (so that $\vartheta(\dot{g}(t))$ is a curve in $\mathfrak{g}$ ). But

$$
\dot{v}(t)=X(v(t))=X\left(\psi_{g(t)}^{M} \check{v}^{\mathrm{H}}(t)\right)=\psi_{g(t)}^{T M} X\left(\check{v}^{\mathrm{H}}(t)\right),
$$

since $X$ is invariant, from which it follows that

$$
\widetilde{\vartheta(\dot{g})} \circ \check{v}^{\mathrm{H}}+\check{v}^{\mathrm{H}}=X \circ \check{v}^{\mathrm{H}} \text {. }
$$

The first term on the left-hand side is vertical, the second horizontal, so this equation is simply the decomposition of $X \circ \check{v}^{\mathrm{H}}$ into its horizontal and vertical components with respect to $\omega$. Thus $g(t)$ must satisfy the so-called reconstruction equation

$$
\vartheta(\dot{g})=\omega\left(X \circ \check{v}^{\mathrm{H}}\right)
$$

where $\omega$ is taken to be the connection 1-form, so that the right-hand side is a curve in $\mathfrak{g}$. This is a differential equation for the curve $g(t)$, and has a unique solution with specified initial value (see for example [14]). Thus the curve $g(t)$ is uniquely determined by the equation and the initial condition $g(0)=e$. Conversely, if $g(t)$ is the solution of the reconstruction equation such that $g(0)=e$ then $v(t)=\psi_{g(t)}^{M} \check{v}^{\mathrm{H}}(t)$ is the integral curve of $X$ through $m$.

We can use this method to obtain an integral curve of $\Gamma$ from one of $\check{\Gamma}$ by using the generalized mechanical connection on $\pi^{T M}: T M \rightarrow T M / G$. The reconstruction equation in this case is

$$
\vartheta(\dot{g})=\Omega^{\mathrm{m}}\left(\Gamma \circ \check{v}^{\mathrm{H}}\right) .
$$

We have therefore to find the vertical component of $\Gamma$ with respect to $\Omega^{\mathrm{m}}$. This is not completely straightforward because we have expressed $\Gamma$ in terms of the invariant basis to obtain the reduced Euler-Lagrange equations in the previous section, whereas $\Omega^{\mathrm{m}}$, as a $\mathfrak{g}$-valued 1-form, is specified by

$$
\Omega^{\mathrm{m}}\left(\tilde{E}_{a}^{\mathrm{C}}\right)=E_{a}, \quad \Omega^{\mathrm{m}}\left(\tilde{E}_{a}^{\mathrm{V}}\right)=0, \quad \Omega^{\mathrm{m}}\left(\bar{X}_{i}^{\mathrm{C}}\right)=0, \quad \Omega^{\mathrm{m}}\left(X_{i}^{\mathrm{V}}\right)=0,
$$

where

$$
\bar{X}_{i}^{\mathrm{C}}=X_{i}^{\mathrm{C}}+B_{i}^{a} \tilde{E}_{a}^{\mathrm{C}}=X_{i}^{\mathrm{C}}-g^{a b} g_{b i} \tilde{E}_{a}^{\mathrm{C}} .
$$

Recall that $\hat{E}_{a}=A_{a}^{b} \tilde{E}_{b}$. From the general properties $(f Z)^{\mathrm{C}}=f Z^{\mathrm{C}}+\dot{f} Z^{\mathrm{v}}$ and $(f Z)^{\mathrm{v}}=f Z^{\mathrm{v}}$ we find that

$$
\hat{E}_{a}^{\mathrm{C}}=A_{a}^{b} \tilde{E}_{b}^{\mathrm{C}}+\dot{A}_{a}^{b} \tilde{E}_{b}^{\mathrm{V}}, \quad \hat{E}_{a}^{\mathrm{V}}=A_{a}^{b} \tilde{E}_{b}^{\mathrm{V}},
$$


and therefore

$$
\Omega^{\mathrm{m}}\left(\hat{E}_{a}^{\mathrm{C}}\right)=A_{a}^{b} E_{b}, \quad \Omega^{\mathrm{m}}\left(\hat{E}_{a}^{\mathrm{V}}\right)=0 .
$$

Moreover, $\Omega^{\mathrm{m}}\left(X_{i}^{\mathrm{C}}\right)=B_{i}^{a} E_{a}$. Thus with

$$
\Gamma=w^{a} \hat{E}_{a}^{\mathrm{C}}+v^{i} X_{i}^{\mathrm{C}}+\Gamma^{a} \hat{E}_{a}^{\mathrm{V}}+\Gamma^{i} X_{i}^{\mathrm{V}} .
$$

we have

$$
\Omega^{\mathrm{m}}(\Gamma)=\left(A_{b}^{a} w^{b}-B_{i}^{a} v^{i}\right) E_{a} .
$$

The reconstruction equation is therefore

$$
\vartheta(\dot{g}(t))=\left(A_{b}^{a}\left(\check{v}^{\mathrm{H}}(t)\right) w^{b}(t)-B_{i}^{a}\left(\check{v}^{\mathrm{H}}(t)\right) v^{i}(t)\right) E_{a} ;
$$

since $w^{a}$ and $v^{i}$ are invariant, $w^{b}(t)$ and $v^{i}(t)$ are just their values on $\check{v}(t)$. In fact in the coordinate system we used in the previous section, corresponding to a chart $U \times G$ on $M$, the (left) action on $M$ is simply given by $\psi_{g}^{M}(x, h)=(x, g h)$, and the induced action on $T M$ by $\psi_{g}^{M}\left(x, h, w^{a}, v^{i}\right)=\left(x, g h, w^{a}, v^{i}\right)$ (to use a somewhat bastardized but self-explanatory notation). We assume that we are able to calculate the integral curve $\check{v}(t)=\left(x(t), w^{a}(t), v^{i}(t)\right)$ of the reduced vector field $\check{\Gamma}$ through $\pi^{T M}\left(v_{0}\right)$ for some point $v_{0} \in T M$. The horizontal lift of $\check{v}$ is a curve in $T M$ of the form $\check{v}^{\mathrm{H}}(t)=\left(x(t), h(t), w^{a}(t), v^{i}(t)\right)$, where $h(t)$ is a curve in $G$ to be determined by $v_{0}$ and by the relation $\Omega^{\mathrm{m}}\left(\dot{v}^{\mathrm{H}}\right)=0$. Moreover, since we have identified the fibres of $\pi^{M}: M \rightarrow M / G$ with $G, A_{b}^{a}$ is effectively a function on $G$. The reconstruction equation can therefore be written

$$
\vartheta(\dot{g}(t))=\left(A_{b}^{a}(h(t)) w^{b}(t)-B_{i}^{a}\left(\check{v}^{\mathrm{H}}(t)\right) v^{i}(t)\right) E_{a},
$$

and the integral curve of $\Gamma$ is just $v(t)=\left(x(t), g(t) h(t), w^{a}(t), v^{i}(t)\right)$.

It may be of interest to express $\Gamma$ in its vertical and horizontal components with respect to $\Omega^{\mathrm{m}}$. In the first place,

$$
\Gamma=\left(A_{b}^{a} w^{b}-B_{i}^{a} v^{i}\right) \tilde{E}_{a}^{\mathrm{C}}+v^{i} \bar{X}_{i}^{\mathrm{C}}+\left(\Gamma^{a}+w^{b} \dot{A}_{b}^{c} \bar{A}_{c}^{a}\right) \hat{E}_{a}^{\mathrm{V}}+\Gamma^{i} X_{i}^{\mathrm{V}},
$$

where $\left(\bar{A}_{a}^{b}\right)$ is the matrix inverse to $\left(A_{a}^{b}\right)$. To proceed further we need a more revealing expression for the term involving $\dot{A}_{b}^{c}$, the total derivative of $A_{b}^{c}$. We can rewrite $\dot{A}_{b}^{c}$ as

$$
w^{d} \hat{E}_{d}\left(A_{b}^{c}\right)+v^{i} X_{i}\left(A_{b}^{c}\right) .
$$

Now

$$
\hat{E}_{d}\left(A_{b}^{c}\right)=A_{d}^{e} \tilde{E}_{e}\left(A_{b}^{c}\right)=A_{d}^{e} C_{e f}^{c} A_{b}^{f},
$$

and therefore

$$
w^{b} w^{d} \hat{E}_{d}\left(A_{b}^{c}\right)=C_{e f}^{c}\left(A_{d}^{e} w^{d}\right)\left(A_{b}^{f} w^{b}\right)=0 .
$$

Recall that $X_{i}\left(A_{b}^{c}\right)=\Upsilon_{i b}^{d} A_{d}^{c}$. Thus

$$
w^{b} \dot{A}_{b}^{c} \bar{A}_{c}^{a}=\Upsilon_{i b}^{a} v^{i} w^{b},
$$

and the coefficient of $\hat{E}_{a}^{\mathrm{V}}$ is $\Gamma^{a}+\Upsilon_{i b}^{a} v^{i} w^{b}$. 
By taking into account the fact that $K_{i j}^{a} v^{i} v^{j}=0$, together with the expression just obtained, it easily follows that the horizontal part of $\Gamma$ projects onto $\check{\Gamma}$, as it must.

The above connection is not the same as the one we have used in [2] for the reconstruction of an arbitrary (not necessarily Lagrangian) second-order field $\Gamma$ on $T M$. There we started with an arbitrary principal connection $\omega$ on $M$ and formed its vertical lift $\Omega$ on $T M$, as we described earlier. For projectable vector fields $W$ on $T M$ we have $\Omega(W)=\omega\left(\tau_{*} W\right)$ (regarding connections as type $(1,1)$ tensor fields). Thus

$$
\Omega\left(\tilde{E}_{a}^{\mathrm{C}}\right)=\tilde{E}_{a}^{\mathrm{C}}, \quad \Omega\left(\tilde{E}_{a}^{\mathrm{V}}\right)=0, \quad \Omega\left(X_{i}^{\mathrm{C}}\right)=0 \quad \text { and } \quad \Omega\left(X_{i}^{\mathrm{V}}\right)=0 .
$$

With respect to the vertical lift connection $\Omega$ the reconstruction equation is just

$$
\vartheta(\dot{g})=A_{a}^{b}(h(t)) w^{b}(t) E_{b},
$$

where the curve $h(t)$ in $G$ is now of course determined by the horizontal lift of $\check{v}$ with respect to $\Omega$, not $\Omega^{\mathrm{m}}$ as before.

Note that in the case of a simple mechanical system, where we can take $\omega$ to be the mechanical connection, the vertical lifts $\Omega$ and $\Omega^{\mathrm{m}}$ coincide. In that case, the Hessian coincides with the Riemannian metric, and the horizontal vector fields $X_{i}$ are orthogonal to the vector fields $\tilde{E}_{a}$, from which $g_{a i}=0$ and therefore also $B_{i}^{a}=0$.

\section{Illustrative examples}

\subsection{A charged particle in a magnetic field and Wong's equations}

We apply the above introduced machinery to the Kaluza-Klein formulation of a charged particle in a magnetic field, see e.g. [10]. We will consider two steps of abstraction. In the first step, we assume given a Riemannian manifold on which a group $G$ acts freely and properly to the left as isometries and we make the further stipulation that the vertical part of the metric (that is, its restriction to the fibres of $\pi^{M}: M \rightarrow M / G$ ) comes from a bi-invariant metric on $G$. We write down the geodesic equations of the metric, by interpreting them as the Euler-Lagrange equations for the kinetic energy Lagrangian. The reduced equations in such a case are known as Wong's equations $[1,13]$. In the second step, we take the manifold to be $\mathrm{E}^{3} \times \mathrm{S}$ and the metric to be of Kaluza-Klein type.

We will denote the metric by $g$. The fact that the symmetry group acts as isometries means that the fundamental vector fields $\tilde{\xi}$ are Killing fields: $\mathcal{L}_{\tilde{\xi}} g=0$. It follows that the components of $g$ with respect to the members of an invariant basis $\left\{\hat{E}_{a}, X_{i}\right\}$ are themselves invariant. We will set $g\left(\hat{E}_{a}, \hat{E}_{b}\right)=h_{a b}$ and $g\left(X_{i}, X_{j}\right)=g_{i j}$. We will use the mechanical connection, which means that $g\left(\hat{E}_{a}, X_{i}\right)=0$. Since both $h_{a b}$ and $g_{i j}$ are $G$ invariant functions, they pass to the quotient; in particular, the $g_{i j}$ are the components with respect to the coordinate fields of a metric on $M / G$, the reduced metric.

The further assumption about the vertical part of the metric has the following implications. It means in the first place that $\mathcal{L}_{\hat{E}_{c}} g\left(\hat{E}_{a}, \hat{E}_{b}\right)=0$ (as well as $\mathcal{L}_{\tilde{E}_{c}} g\left(\hat{E}_{a}, \hat{E}_{b}\right)=$ 0 ), and secondly that the $h_{a b}$ must be independent of the coordinates $x^{i}$ on $M / G$, 
which is to say that they must be constants. From the first condition, taking into account the bracket relations $\left[\hat{E}_{a}, \hat{E}_{b}\right]=C_{a b}^{c} \hat{E}_{c}$, we easily find that the $h_{a b}$ must satisfy $h_{a d} C_{b c}^{d}+h_{b d} C_{a c}^{d}=0$. Recall that if we set

$$
X_{i}=\frac{\partial}{\partial x^{i}}-\gamma_{i}^{a} \hat{E}_{a}
$$

for some $G$-invariant coefficients $\gamma_{i}^{a}$, we get $\Upsilon_{i a}^{b}=\gamma_{i}^{c} C_{a c}^{b}$, and therefore $h_{a c} \Upsilon_{i b}^{c}+h_{b c} \Upsilon_{i b}^{c}=$ 0 .

The geodesic equations may be derived from the Lagrangian

$$
L=\frac{1}{2} g_{\alpha \beta} u^{\alpha} u^{\beta}=\frac{1}{2} g_{i j} v^{i} v^{j}+\frac{1}{2} h_{a b} w^{a} w^{b} .
$$

It is of course $G$-invariant. We may therefore apply Lagrange-Poincaré reduction, which gives the reduced equations

$$
\begin{aligned}
\frac{d}{d t}\left(g_{i j} v^{j}\right)-\frac{1}{2} \frac{\partial g_{j k}}{\partial x^{i}} v^{j} v^{k} & =-\left(K_{i j}^{a} v^{j}+\Upsilon_{i b}^{a} w^{b}\right) h_{a c} w^{c} \\
\frac{d}{d t}\left(h_{a b} w^{b}\right) & =\left(\Upsilon_{i a}^{b} v^{i}+C_{a c}^{b} w^{c}\right) h_{b d} w^{d} .
\end{aligned}
$$

Now $\Upsilon_{i b}^{a} h_{a c}$ is skew-symmetric in $b$ and $c$, and $C_{a c}^{b} h_{b d}$ is skew-symmetric in $c$ and $d$, so the final terms in each equation vanish identically. Let $\Gamma_{j k}^{i}$ be the connection coefficients of the Levi-Civita connection of the reduced metric $g_{i j}$. Then, we may write the equations in the form

$$
\begin{aligned}
& g_{i j}\left(\ddot{x}^{j}+\Gamma_{k l}^{j} \dot{x}^{k} \dot{x}^{l}\right)=-h_{b c} K_{i j}^{c} \dot{x}^{j} w^{b} \\
& h_{a b}\left(\dot{w}^{b}+\Upsilon_{i c}^{b} \dot{x}^{i} w^{c}\right)=0,
\end{aligned}
$$

using the skew-symmetry of $\Upsilon_{i b}^{c} h_{a c}$ again in the second equation. Given that $K_{i j}^{c}$ is of course skew-symmetric in its lower indices, these equations are equivalent to

$$
\begin{aligned}
\ddot{x}^{i}+\Gamma_{j k}^{i} \dot{x}^{j} \dot{x}^{k} & =g^{i k} h_{b c} K_{j k}^{c} \dot{x}^{j} w^{b} \\
\dot{w}^{a}+\Upsilon_{j b}^{a} \dot{x}^{j} w^{b} & =0 .
\end{aligned}
$$

These are Wong's equations.

Let us now take $M$ to be $\mathrm{E}^{3} \times \mathrm{S}$, with coordinates $\left(x^{i}, \theta\right)$. Let $A_{i}$ be the components of a covector field on $\mathrm{E}^{3}$, and define a metric $g$ on $M$, the Kaluza-Klein metric, by

$$
g=\delta_{i j} d x^{i} \odot d x^{j}+\left(A_{i} d x^{i}+d \theta\right)^{2}
$$

where $\left(\delta_{i j}\right)$ is the Euclidean metric. The Kaluza-Klein metric admits the Killing field $E=\partial / \partial \theta$. The vector fields $X_{i}=\partial / \partial x^{i}-A_{i} \partial / \partial \theta$ are orthogonal to $E$ and invariant; moreover $g_{i j}=g\left(X_{i}, X_{j}\right)=\delta_{i j}$, while $g(E, E)=1$. Finally

$$
\left[X_{i}, X_{j}\right]=\left(\frac{\partial A_{i}}{\partial x^{j}}-\frac{\partial A_{j}}{\partial x^{i}}\right) \frac{\partial}{\partial \theta} .
$$

Putting these values into the reduced equations above we obtain

$$
\ddot{x}^{i}=w \dot{x}^{j}\left(\frac{\partial A_{i}}{\partial x^{j}}-\frac{\partial A_{j}}{\partial x^{i}}\right), \quad \dot{w}=0 .
$$

These are the equations of motion of a particle of unit mass and charge $w$ in a magnetic field whose vector potential is $A_{i} d x^{i}$. 


\subsection{A worked-out example}

We will consider the Lie group $G$ of the affine line. An element of this group is an affine map $\mathbf{R} \rightarrow \mathbf{R}: t \mapsto \exp (\theta) t+\phi$ and can be represented by the matrix

$$
\left(\begin{array}{ll}
\exp \theta & \phi \\
0 & 1
\end{array}\right)
$$

The identity element is just $t \mapsto t$ (the identity matrix) and multiplication on the left of $\left(\theta_{2}, \phi_{2}\right)$ by $\left(\theta_{1}, \phi_{1}\right)$ is given by the composition of the two affine maps, i.e. the element

$$
\left(\theta_{1}, \phi_{1}\right) *\left(\theta_{2}, \phi_{2}\right)=\left(\theta_{1}+\theta_{2}, \exp \left(\theta_{1}\right) \phi_{2}+\phi_{1}\right) .
$$

The corresponding Lie algebra is given by the set of matrices of the form

$$
\left(\begin{array}{ll}
a & b \\
0 & 0
\end{array}\right)
$$

The manifold $M$ of interest is $G \times \mathbf{R}$. The action on the manifold is given by left translation on the $G$ factor of $G \times \mathbf{R}$. We will write $x^{0}=x$ for the coordinate on $\mathbf{R}$. For this action and this manifold there is a trivial principal connection $\omega$, with $\gamma_{a}^{0}=0$. A basis of fundamental vector fields is

$$
\tilde{E}_{1}=\frac{\partial}{\partial \theta}+\phi \frac{\partial}{\partial \phi}, \quad \tilde{E}_{2}=\frac{\partial}{\partial \phi} .
$$

The vector fields that are horizontal with respect to the trivial connection all lie in the direction of $X=\partial / \partial x$. The adapted coordinates $\left(v^{i}, v^{a}\right)$ are therefore $v^{0}=\dot{x}$ and $v^{1}=\dot{\theta}, v^{2}=\dot{\phi}-\phi \dot{\theta}$.

The Lie algebra bracket is given by $\left[\tilde{E}_{1}, \tilde{E}_{2}\right]=-\tilde{E}_{2}$. The complete and vertical lifts of this basis are

$$
\tilde{E}_{1}^{\mathrm{C}}=\frac{\partial}{\partial \theta}+\phi \frac{\partial}{\partial \phi}+\dot{\phi} \frac{\partial}{\partial \dot{\phi}}, \quad \tilde{E}_{2}^{\mathrm{C}}=\frac{\partial}{\partial \phi}, \quad \tilde{E}_{1}^{\mathrm{V}}=\frac{\partial}{\partial \dot{\theta}}+\phi \frac{\partial}{\partial \dot{\phi}}, \quad \tilde{E}_{2}^{\mathrm{V}}=\frac{\partial}{\partial \dot{\phi}} .
$$

An invariant basis of vector fields is given by $\left\{\hat{E}_{1}, \hat{E}_{2}, X\right\}$, where

$$
\hat{E}_{1}=\frac{\partial}{\partial \theta}, \quad \hat{E}_{2}=\exp (\theta) \frac{\partial}{\partial \phi}
$$

and the coordinates with respect to this basis are $v^{0}=\dot{x}, w_{1}=\dot{\theta}$ and $w_{2}=\exp (-\theta) \dot{\phi}$. The complete and vertical lifts of the above basis are

$$
\begin{aligned}
& \hat{E}_{1}^{\mathrm{C}}=\frac{\partial}{\partial \theta}, \quad \hat{E}_{2}^{\mathrm{C}}=\exp (\theta)\left(\frac{\partial}{\partial \phi}+\dot{\theta} \frac{\partial}{\partial \dot{\phi}}\right), \quad \hat{E}_{1}^{\mathrm{V}}=\frac{\partial}{\partial \dot{\theta}}, \quad \hat{E}_{2}^{\mathrm{V}}=\exp (\theta) \frac{\partial}{\partial \dot{\phi}}, \\
& X^{\mathrm{C}}=\frac{\partial}{\partial x}, \quad X^{\mathrm{V}}=\frac{\partial}{\partial \dot{x}} .
\end{aligned}
$$

Finally, the matrix $\mathcal{A}$, defined by the relation $\hat{E}_{a}(x, g)=A_{a}^{b}(g) \tilde{E}_{b}(x, g)$, is here

$$
A(g)=\left(\begin{array}{cc}
1 & 0 \\
-\phi & \exp (\theta)
\end{array}\right)
$$

At the identity of the Lie group, the matrix $A$ is the identity matrix, as it should be. 
If we use the invariant fibre coordinates $\left(v^{0}, w^{a}\right)$, the induced action on $T M$ is simply $\psi_{\left(\phi_{1}, \theta_{1}\right)}^{T M}\left(x,(\phi, \theta), \dot{x}, w_{1}, w_{2}\right)=\left(x,\left(\phi_{1}, \theta_{1}\right) *(\phi, \theta), \dot{x}, w_{1}, w_{2}\right)$. Since the coordinates $\left(x, \dot{x}, w_{1}, w_{2}\right)$ can be interpreted as coordinates on $T M / G=T \mathbf{R} \times T G / G=T \mathbf{R} \times \mathfrak{g}$, invariance of the Lagrangian simply means that the group variables do not explicitly appear in the Lagrangian, when it is written in terms of the invariant fibre coordinates. We will work with the Lagrangian

$$
L=\frac{1}{2} \dot{\theta}^{2}+q \dot{x} \dot{\theta}+\frac{1}{2} \dot{x}^{2}+\ln (\exp (-\theta) \dot{\phi}),
$$

where $q$ is a constant. The Lagrangian is clearly not of the simple type. One easily verifies that the Lagrangian is invariant: $\tilde{E}_{1}^{\mathrm{C}}(L)=0=\tilde{E}_{2}^{\mathrm{C}}(L)$. In the invariant fibre coordinates $\left(w_{i}=\left(w_{1}, w_{2}\right), \dot{x}\right)$, the Lagrangian is

$$
L=\frac{1}{2} w_{1}^{2}+q \dot{x} w_{1}+\frac{1}{2} \dot{x}^{2}+\ln \left(w_{2}\right),
$$

so, indeed, $(\phi, \theta)$ do not appear explicitly. This Lagrangian is also invariant under the obvious $\mathbf{R}$-action on the manifold, but we will not take this into consideration.

The Hessian matrix in the basis $\left\{\tilde{E}_{a}, X\right\}$ is here

$$
g=\left(\begin{array}{ccc}
1-\frac{\phi^{2}}{\dot{\phi}^{2}} & -\frac{\phi}{\dot{\phi}^{2}} & q \\
-\frac{\phi}{\dot{\phi}^{2}} & -\frac{1}{\phi^{2}} & 0 \\
q & 0 & 1
\end{array}\right)
$$

The determinant of $g$ is $\left(q^{2}-1\right) / \dot{\phi}^{2}$, so the Lagrangian is regular as long as $q^{2} \neq 1$. The upper left $(2,2)$ matrix represents $\left(g_{a b}\right)$. It is non-singular since its determinant is $-1 / \dot{\phi}^{2}$. Its inverse is

$$
\left(g^{a b}\right)=\left(\begin{array}{cc}
1 & -\phi \\
-\phi & \phi^{2}-\dot{\phi}^{2}
\end{array}\right) .
$$

The vector field $\bar{X}$ along $\tau$ that projects onto $\partial / \partial x$ on $M / G$ and is horizontal for the generalized mechanical connection $\omega^{\mathrm{m}}$ is

$$
\begin{aligned}
\bar{X} & =\frac{\partial}{\partial x}-g^{b c} g_{c x} \tilde{E}_{b} \\
& =\frac{\partial}{\partial x}-q \tilde{E}_{1}+q \phi \tilde{E}_{2} \\
& =\frac{\partial}{\partial x}-q \frac{\partial}{\partial \theta} .
\end{aligned}
$$

This vector field is in fact a basic vector field along $\tau$. Although the Lagrangian and the Hessian are not of the simple type, in this example the generalized mechanical connection turns out to be derived from a principal connection on $M \rightarrow M / G$, and is not of the most general case of an invariant connection on the pullback bundle.

The corresponding vector field that is horizontal with respect to the connection $\Omega^{\mathrm{m}}$ is

$$
\begin{aligned}
\bar{X}^{\mathrm{C}} & =\frac{\partial}{\partial x}-q \tilde{E}_{1}^{\mathrm{C}}+q \phi \tilde{E}_{2}^{\mathrm{C}} \\
& =\frac{\partial}{\partial x}-q \frac{\partial}{\partial \theta}-q \dot{\phi} \frac{\partial}{\partial \dot{\phi}}
\end{aligned}
$$


Let us look now at the dynamics. First, let us pretend that we do not know that the system exhibits symmetry and solve directly the Euler-Lagrangian equations for this Lagrangian. The equations for $x, \theta$ and $\phi$ are here, respectively:

$$
q \ddot{\theta}+\ddot{x}=0, \quad \ddot{\theta}+q \ddot{x}+1=0, \quad-\frac{\ddot{\phi}}{\dot{\phi}^{2}}=0 .
$$

From the $x$-equation, it is again clear that there is also $\mathbf{R}$-symmetry. The solution of the system is easy to find. With the obvious notations for the integration constants, we obtain

$$
x(t)=-\frac{1}{2} \frac{q t^{2}}{q^{2}-1}+\dot{x}_{0} t+x_{0}, \quad \theta(t)=\frac{1}{2} \frac{t^{2}}{q^{2}-1}+\dot{\theta}_{0} t+\theta_{0}, \quad \phi(t)=\dot{\phi}_{0} t+\phi_{0} .
$$

We will assume that $\dot{\phi}_{0}>0$.

We now apply the technique of symmetry reduction and reconstruction. We first need a solution of the Lagrange-Poincaré equations. Since for the current example the connection coefficients of the trivial connection vanish, these equations become

$$
\frac{d}{d t}\left(\frac{\partial l}{\partial w^{b}}\right)=\frac{\partial l}{\partial w^{a}} C_{b d}^{a} w^{d}, \quad \frac{d}{d t}\left(\frac{\partial l}{\partial \dot{x}}\right)-\frac{\partial l}{\partial x}=0 .
$$

The reduced Lagrangian on $T M / G$ is

$$
l\left(x, \dot{x}, w_{1}, w_{2}\right)=\frac{1}{2} w_{1}^{2}+q \dot{x} w_{1}+\frac{1}{2} \dot{x}^{2}+\ln \left(w_{2}\right),
$$

and the reduced equations are

$$
\dot{w}_{1}+q \ddot{x}=-1, \quad \dot{w}_{2}=-w_{1} w_{2}, \quad q \dot{w}_{1}+\ddot{x}=0 .
$$

If we set $w_{1}(0)=\dot{\theta}_{0}$ and $w_{2}(0)=\exp \left(-\theta_{0}\right) \dot{\phi}_{0}$, the solution of the above equations is

$$
\begin{aligned}
& x(t)=-\frac{1}{2} \frac{q t^{2}}{q^{2}-1}+\dot{x}_{0} t+x_{0}, \quad w_{1}(t)=\frac{t}{q^{2}-1}+\dot{\theta}_{0}, \\
& w_{2}(t)=\dot{\phi}_{0} \exp \left(-\theta_{0}-\frac{1}{q^{2}-1}\left(\frac{1}{2} t^{2}-\dot{\theta}_{0} t+\dot{\theta}_{0} q^{2} t\right)\right) .
\end{aligned}
$$

Clearly, $x(t)$ has the desired form. From the reduced solution $\check{v}(t)=$ $\left(x(t), \dot{x}(t), w_{1}(t), w_{2}(t)\right)$, we could determine the remaining coordinates $(\theta(t), \phi(t))$ directly from the relations $\dot{\theta}=w_{1}$ and $\exp (-\theta) \dot{\phi}-\dot{\theta}=w_{2}$. The reconstruction process as described above splits this calculation into two steps: first we calculate the horizontal lift of $\check{v}(t)$, and then we use it in the reconstruction equation. In this way, we will see the effect of changing the connection in the reconstruction equation.

The coordinates of the horizontal lift using the generalized mechanical connection are $\check{v}^{\mathrm{H}}(t)=\left(x(t), \phi^{\mathrm{H}}(t), \theta^{\mathrm{H}}(t), \dot{x}(t), w_{1}(t), w_{2}(t)\right)$, with respect to the invariant basis. They can be determined by the relation

$$
0=\Omega^{\mathrm{m}}\left(\check{v}^{\mathrm{H}}(t)\right)=\omega^{\mathrm{m}}\left(\check{v}^{\mathrm{H}}, T \tau \circ \check{v}^{\mathrm{H}}\right)=\omega^{\mathrm{m}}\left(\check{v}^{\mathrm{H}}, \frac{d}{d t}\left(\tau \circ \check{v}^{\mathrm{H}}\right)\right)
$$

This equation for $\tau \circ \check{v}^{\mathrm{H}}(t)=\left(x(t), \phi^{\mathrm{H}}(t), \theta^{\mathrm{H}}(t)\right)$ is

$$
\dot{\theta}^{\mathrm{H}}=-q \dot{x}, \quad \dot{\phi}^{\mathrm{H}}=-q \dot{x} \phi^{\mathrm{H}}+q \dot{x} \phi^{\mathrm{H}}=0 .
$$


Therefore $\theta^{\mathrm{H}}(t)=-q x(t)+q x_{0}+\theta_{0}$ and $\phi^{\mathrm{H}}(t)=\phi_{0}$.

Now we determine the curve $g(t)=\left(\theta_{1}(t), \phi_{1}(t)\right)$ in $G$ such that $v=g \check{v}^{\mathrm{H}}$ is the solution of the Euler-Lagrange equations with the given initial values. This curve is the solution through the identity of the reconstruction equation $g^{-1} \dot{g}=\Omega^{\mathrm{m}}\left(\Gamma \circ \check{v}^{\mathrm{H}}\right)$. The left hand side is $\dot{\theta}_{1} E_{1}+\exp \left(-\theta_{1}\right) \dot{\phi}_{1} E_{2}$. The right hand side is $\omega^{\mathrm{m}}\left(\check{v}^{\mathrm{H}}\right)$, or $\left(w_{1}+q \dot{x}\right) \tilde{E}_{1}^{\mathrm{C}} \circ \check{v}^{\mathrm{H}}+\left(w_{2}+q \phi_{m} \dot{x}\right) \tilde{E}_{1}^{\mathrm{C}} \circ \check{v}^{\mathrm{H}}$. The reconstruction equations are therefore

$$
\dot{\theta}_{1}=w_{1}+q \dot{x}, \quad \exp \left(-\theta_{1}\right) \dot{\phi}_{1}=-\phi^{\mathrm{H}} w_{1}+\exp \left(\theta_{m}\right) w_{2}-q \phi^{\mathrm{H}} \dot{x} .
$$

Solving the above equations for $\left(\theta_{1}, \phi_{1}\right)$ gives

$$
\theta_{1}(t)=-\frac{1}{2} t^{2}+\left(q \dot{x}_{0}+\dot{\theta}_{0}\right) t, \quad \phi_{1}(t)=\dot{\phi}_{0} t+\phi_{0}\left(1-\exp \left(\frac{1}{2}\left(2 q \dot{x}_{0}-t+2 \dot{\theta}_{0}\right) t\right)\right) .
$$

The final solution is therefore indeed

$$
\theta(t)=\theta_{1}(t)+\theta_{m}(t)=\frac{1}{2} \frac{t^{2}}{q^{2}-1}+\dot{\theta}_{0} t+\theta_{0}, \quad \phi(t)=\exp \left(\theta_{1}(t)\right) \phi_{m}(t)+\phi_{1}(t)=\dot{\phi}_{0} t+\phi_{0} .
$$

If we use the vertical lift $\Omega$ of the trivial (principal) connection $\omega$, the equation that determines the horizontal lift $\check{v}^{\mathrm{H}}\left(\right.$ again with group coordinates $\left.\left(\phi^{\mathrm{H}}, \theta^{\mathrm{H}}\right)\right)$ is $\omega\left(\frac{d}{d t}\left(\tau \circ \check{v}^{\mathrm{H}}\right)\right)=$ 0 , or

$$
\dot{\theta}^{\mathrm{H}}=0, \quad \dot{\phi}^{\mathrm{H}}=0,
$$

from which $\theta^{\mathrm{H}}(t)=\theta_{0}$ and $\phi^{\mathrm{H}}(t)=\phi_{0}$. The equation $g^{-1} \dot{g}=\Omega\left(\Gamma \circ \check{v}^{\mathrm{H}}\right)=\omega\left(\check{v}^{\mathrm{H}}\right)$ for $g=\left(\phi_{1}(t), \theta_{1}(t)\right)$ is then

$$
\dot{\theta}_{1}=w_{1}, \quad \exp \left(-\theta_{1}\right) \dot{\phi}_{1}=-\phi^{\mathrm{H}} w_{1}+\exp \left(\theta^{\mathrm{H}}\right) w_{2} .
$$

Its solution is

$$
\theta_{1}(t)=\frac{1}{2} \frac{t^{2}}{q^{2}-1}+\dot{\theta}_{0} t, \quad \phi_{1}(t)=\phi_{0}\left(1-\exp \left(\frac{1}{2} \frac{t^{2}-2 \dot{\theta}_{0} t+2 \dot{\theta}_{0} q^{2} t}{q^{2}-1}\right)\right)+\dot{\phi}_{0} t
$$

which leads again to the same solution $(\theta(t), \phi(t))$.

The solution using the vertical lift of $\omega$ is somewhat simpler, but this is only to be expected since $\omega$ is trivial.

\section{Conclusions and Outlook}

We have considered regular Lagrangians that are invariant under a symmetry Lie group and we have derived the reduced Euler-Lagrange equations, the so-called LagrangePoincaré equations. Our framework relied on the associated Euler-Lagrange vector field and its quotient field, rather than on the variational formalism and on the use of well-chosen quasi-velocities. Given an integral curve of the reduced vector field, we have shown how to reconstruct an integral curve of the original Euler-Lagrange field by means of a principal connection that is natural associated to the Lagrangian, the so-called generalized mechanical connection.

In forthcoming papers, we will apply the same technique also to the context of a different but related Lagrangian reduction technique [3] and to the characterization of relative equilibria [4]. 


\section{Acknowledgments}

The first author is currently a Research Fellow at The University of Michigan through a Marie Curie Fellowship. He is grateful to the Department of Mathematics for its hospitality. He also acknowledges a research grant (Krediet aan Navorsers) from the Fund for Scientific Research - Flanders (FWO-Vlaanderen), where he is an Honorary Postdoctoral Fellow.

The second author is a Guest Professor at Ghent University: he is grateful to the Department of Mathematical Physics and Astronomy at Ghent for its hospitality.

\section{References}

[1] H. Cendra, J.E. Marsden and T.S. Ratiu, Lagrangian Reduction by Stages, Memoirs of the American Mathematical Society 152, AMS 2001.

[2] M. Crampin and T. Mestdag, Reduction and reconstruction aspects of second-order dynamical systems with symmetry, preprint (2006), available at maphyast.ugent.be Theoretical Mechanics.

[3] M. Crampin and T. Mestdag, Routh's procedure for non-Abelian symmetry groups, J. Math. Phys. (2008), to appear.

[4] M. Crampin and T. Mestdag, Relative equilibria of Lagrangian systems with symmetry, preprint (2007), available at maphyast.ugent.be Theoretical Mechanics.

[5] M. Crampin and F.A.E. Pirani, Applicable Differential Geometry, LMS Lecture Notes 59, Cambridge University Press 1988.

[6] M. Crampin and D. J. Saunders, Affine and projective transformations of Berwald connections, Diff. Geom. Appl. 25 (2007) 235-250.

[7] S. Kobayashi and K. Nomizu, Foundations of Differential Geometry, Vol I, Wiley 1963.

[8] J. E. Marsden, G. Misiolek, J-P. Ortega, M. Perlmutter and T. S. Ratiu, Hamiltonian Reduction by Stages, Lecture Notes in Mathematics 1913, Springer 2007.

[9] D. Lewis, Lagrangian block diagonalization, J. Dynamics and Diff. Equations 4 (1992) 1-41.

[10] J.E. Marsden and T. Ratiu, Introduction to Mechanics and Symmetry, Texts in Applied Mathematics 17, Springer 1999.

[11] J.E. Marsden, T. Ratiu and J. Scheurle, Reduction theory and the Lagrange-Routh equations, J. Math. Phys. 41 (2000) 3379-3429.

[12] E. Martínez, J.F. Cariñena and W. Sarlet, Derivations of differential forms along the tangent bundle projection, Diff. Geom. Appl. 2 (1992) 17-43.

[13] R. Montgomery, Canonical formulations of a classical particle in a Yang-Mills field and Wong's equations, Lett. Math. Phys. 8 (1984), 59-67.

[14] R. W. Sharpe, Differential Geometry, Graduate Texts in Mathematics 166, Springer 1997.

[15] J. Szilasi, A setting for spray and Finsler geometry, in: Handbook of Finsler Geometry (ed. P. L. Antonelli), Kluwer 2003, 1183-1426. 